\title{
Risk based bridge data collection and asset management and the role of structural health monitoring
}

\author{
Piotr Omenzetter ${ }^{*}$, Simon Bush ${ }^{\mathrm{ab}}$, Theunis Henning ${ }^{\mathrm{a}}$, Peter McCarten ${ }^{\mathrm{c}}$ \\ ${ }^{\text {a }}$ The University of Auckland, Private Bag 92019, Auckland, New Zealand; \\ ${ }^{\mathrm{b}}$ Opus International Consultants, Auckland, PO Box 5848, New Zealand; \\ 'Opus International Consultants, Napier 4110, 6 Ossian St, New Zealand
}

\begin{abstract}
Bridges are critical to the operation and functionality of the whole road networks. It is therefore essential that specific data is collected regarding bridge asset condition and performance, as this allows proactive management of the assets and associated risks and more accurate short and long term financial planning. This paper proposes and discusses a strategy for collection of data on bridge condition and performance. Recognizing that risk management is the primary driver of asset management, the proposed strategy prioritizes bridges for levels of data collection including core, intermediate and advanced. Individual bridges are seen as parts of wider networks and bridge risk and criticality assessment emphasizes bridge failure or underperformance risk in the network context. The paper demonstrates how more reliable and detailed data can assist in managing network and bridge risks and provides a rationale for application of higher data collection levels for bridges characterized by higher risk and criticality. As the bridge risk and/or criticality increases planned and proactive integration of structural health monitoring (SHM) data into asset management is outlined. An example of bridge prioritization for data collection using several bridges taken from a national highway network is provided using an existing risk and criticality scoring methodology. The paper concludes with a discussion on the role of SHM in data collection for bridge asset management and where SHM can make the largest impacts.
\end{abstract}

Keywords: Bridges, infrastructure asset management, data collection, risk, criticality, structural health monitoring

\section{INTRODUCTION}

Structural health monitoring (SHM) has been defined by Aktan et al. ${ }^{1}$ as " a process involving tracking any aspect of structural performance or health by measuring data and interpreting them in conjunction with application-specific knowledge so that structural condition and reliability can be quantified objectively." Over the past two decades or so, considerable research has been conducted and marked progress achieved across the many facets of SHM, including sensor and system development, data storage and transmission, and data interrogation and processing for extracting information about structural performance and reliability.

Inaudi and Walder ${ }^{2}$ enumerate a number of cases where SHM is recommended and/or required:

- New structures with innovative design, construction techniques, or materials,

- New structures with unusual risks, including geological, seismic, meteorological, environment, construction, and quality risk,

- New or existing structures which are representative of a larger population of similar structures, where SHM derived information can be extrapolated to the wider population,

- New or existing structures that are critical at a network level, such that their failure or deficiency would have a serious impact on the network functioning,

- Existing structures with known deficiencies, problems and/or very low rating, and

- Candidate structures for replacement or refurbishment works, where the real need for interventions can be assessed a priori and repair efficiency evaluated a posteriori.

*p.omenzetter@auckland.ac.nz; phone 64 9923-8138; fax 64 9373-7462; www.auckland.ac.nz 
Examining the above list for examples of SHM applications, one can find well publicized cases of planned and proactive integration of SHM into newly constructed innovative, landmark, record breaking and/or exotic structures ${ }^{3}$. Such SHM projects are often driven by academic and research interests, the push to expand structural design guidelines and validate daring design solutions. Down the above list, SHM applications are envisaged to existing, potentially numerous, structures that are increasingly in the domain of interest of infrastructure asset managers rather than structural designers. Despite the existing examples of successful applications of SHM to such cases ${ }^{4}$, it can be argued that most of such projects are ad hoc in their nature and reactive to existing identified problems rather than proactive, are not well integrated into the overall asset management planning, and their benefits are not always clearly demonstrated.

Potential benefits of using SHM are often summarized as follows²:

- Reducing uncertainty about structural condition and performance,

- Discovering hidden structural reserves,

- Discovering deficiencies that may be missed by traditional assessment techniques

- Increasing safety and reliability,

- Ensuring long term quality of aging infrastructure,

- Allowing better informed asset management, and

- Increasing knowledge about in-situ structural performance.

The above list, examined from the vintage point of the infrastructure asset manager, makes SHM a very desirable tool as these potential benefits are addressing the key challenges of asset management, i.e., the need for advanced knowledge about asset condition and performance, and reliable data for planning to ensure that assets are performing to the expected level well into the future. In spite of that, SHM has made only limited transition from the research domain into widespread practical applications. In order to achieve a widespread, planned and proactive integration of SHM into infrastructure asset management and realize its potential benefits it is necessary to understand the key drivers of asset management. By achieving this understanding, SHM can be strategically employed to enhance the asset management processes and address its current shortcomings in a cost effective way.

Bridges are important, often critical and expensive components of infrastructure systems and transportation networks and hence require particular attention of transportation asset managers. From a technical point of view, they lend themselves, arguably more naturally than other structures, to SHM. One of the reasons is that, despite being often structurally complex, they have a relatively less non-structural components and services, e.g., compared to buildings. Because of these reasons, this paper examines the use of SHM in the context of bridge structures as parts of transportation networks.

The aims of this discussion paper are: i) to highlight managing risk and criticality as the major driver of asset management, ii) to demonstrate how informed asset management decisions must rely on high quality data about failure probabilities and their consequences, iii) to present a risk and criticality based strategy for prioritization of bridges for targeted data collection, iv) to show how SHM can help in providing better data and be integrated into managing risks, and iv) to discuss the role of SHM in bridge data collection and identify the areas where it can make the largest impacts. The remainder of the paper is divided into four parts, each addressing one the objectives.

\section{RISK AND CRITICALITY AS DRIVERS OF ASSET MANAGEMENT}

A well recognized standard that covers general asset management is PAS 55-1:2008: Specification for the Optimized Management of Physical Assets. ${ }^{5}$. The document defines asset management as "systematic and coordinated activities and practices through which an organization optimally and sustainably manages its assets and asset systems, their associated performance, risks and expenditures over their life cycles for the purpose of achieving its organizational strategic plan". It is clear from the above definition that at the core of asset management there is risk management ${ }^{6}$ and so it makes sense that SHM be promoted as a tool to effectively address risk management, including risk assessment, mitigation and reduction.

The commonly accepted definition of risk, $R$, is the probability, $P(F)$, of a certain undesirable event $F$, such as failure, multiplied by the expected value of consequences, $\mathrm{E}[C \mid F]$, given the event occurs: 


$$
R=P(F) \times \mathrm{E}[C \mid F]
$$

This notion of risk is used throughout this study. "Failure" is here understood broadly as any situation when a bridge does not fulfill its expected target performance. This may, in extreme and rare cases, be the same as structural collapse or damage but also includes non-catastrophic failures where, e.g., vehicle load or speed are restricted to manage structural element fatigue ${ }^{7}$. Failure consequences, or impacts, can be given in financial terms, number of fatalities or similar ${ }^{6}$. As far as financial consequences are concerned these are considered at the level of a given transportation network to which the bridge in question belongs, and include not only the direct repair, strengthening or replacement costs but also the costs due to potential traffic disruption and delays, the need to prepare and operate alternative routes, etc.

The way risk information is presented has potentially important consequences for risk perception. There is arguably a higher degree of tolerance for "unavoidable" infrastructure failures due to natural causes compared to "man made" causes, e.g., due to a design error or inappropriate maintenance ${ }^{8}$. Given that according to Matousek and Schneider" (as reported by Stewart and Melchers ${ }^{10}$ ) many a structural failure are due to human error such as design code violations or construction errors this distinction may be important in risk analysis. Society also appears to be much more concerned with sudden, one off events with large consequences, e.g., a major natural disaster with severe damage to infrastructure or bridge collapses with fatalities, but accepts more easily series of frequent, smaller failures even though their cumulative effects over time can be comparable. In other words, risk tolerance decreases as the consequences increase irrespective of the occurrence probabilities involved ${ }^{6}$. In the context of transportation networks and bridge asset management, bridges with large consequence of failure will be herein referred to as "critical". Potential failure consequences are the measure of criticality. Thus, it seems appropriate to manage bridge risk in the two dimensional, risk-criticality domain. This is an approach that takes a wider view of bridges as parts of transportation networks, and differs from studies where bridge data collection and SHM integration were approached from the point of view of managing reliabilities of individual structures, see, e.g., Frangopol et al. ${ }^{11}$

As obviously transportation asset managers have limited, often tight, budgets, any risk management and/or mitigation decisions and actions including strengthening or replacement of bridges must take into account their associated costs. The same must be done when deciding which approaches to bridge data collection are to be adopted as different regimes and techniques will require different resources. For example, adopting SHM may incur additional costs compared to visual inspections, and there will also be differences between the costs and benefits of periodic and continuous monitoring ${ }^{12}$.

\section{NETWORK RISK ANALYSIS AND ITS IMPLICATIONS FOR BRIDGE DATA COLLECTION}

Risk based decision analysis can be presented in a generic format ${ }^{13}$ as shown in Figure 1. This chapter discusses how the process can be adopted for bridge asset management. For the purpose of our current considerations a transportation network of roads including $N$ bridges is considered. An example of such a network comprising Bridge \#1 through to Bridge $\# N$ is shown in Figure 2. This network example is used only to focus the discussion by providing a convenient illustration for the concepts introduced but is not intended to be a model of any actual network.

Failure of each bridge can happen in a number of ways related to the exceedance of different limit states, e.g., Moon et $\mathrm{al}^{8}{ }^{8}$ consider broadly structural safety limit state, hydraulic/geotechnical safety limit state, serviceability, durability and maintenance limit state and functionality limit state. Consider there are $M$ possible limit states. Denote by $F_{n, m}(n=1,2$, $\ldots, N, m=1,2, \ldots, M)$ an event that Bridge $\# N$ fails the $M$-th limit state, and by $\sim F_{n, \mathrm{~m}}$ the event that it does not. Each of such events can be assigned a probability of occurrence, $P\left(F_{n, m}\right)$. These can be calculated from the structural reliability theory ${ }^{14}$ for each bridge and limit state as:

$$
P(F)=P(R-S \leq 0)=\int_{-\infty}^{+\infty} \int_{-\infty}^{s \geq r} f_{r}(r) f_{s}(s) d r d s=\int_{-\infty}^{+\infty} F_{r}(x) f_{s}(x) d x
$$




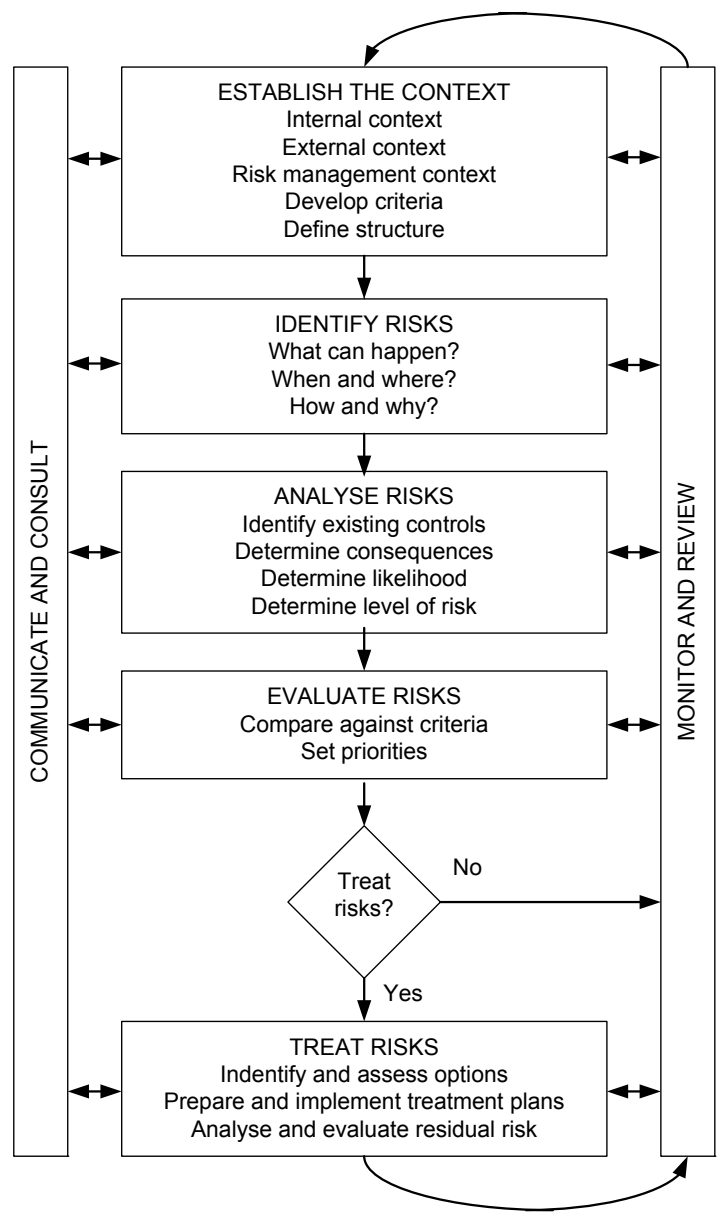

Figure 1. Overview of risk management process $\left(\operatorname{after}^{13}\right)$

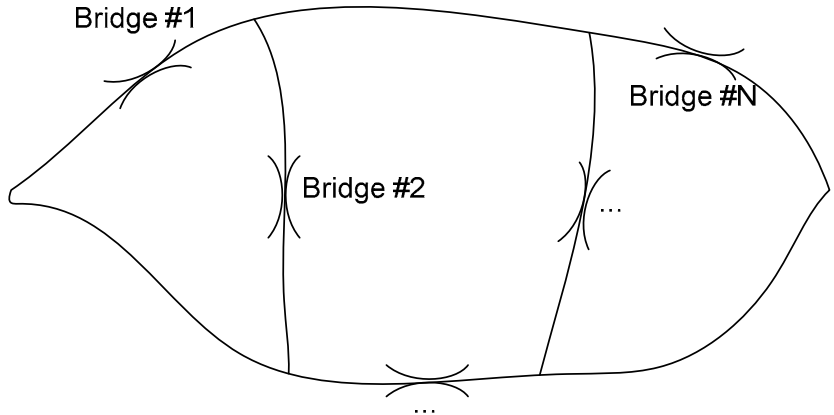

Figure 2. Example of a transportation network with bridges.

where in Eq. (2), $R$ is the structure capacity, $S$ demand resultant, $f_{r}$ probability density function of capacity, $f_{s}$ probability density function of demand resultant, and $F_{r}$ cumulative probability function of capacity, respectively. Implicit in Eq. (2) is the assumption that failure probabilities for different limit states are independent, but there are well known generalizations $^{14}$ of Eq. (2) if this assumption does not hold. It is also noted that failure probabilities are typically time dependent. This is a consequence of structural capacity diminishing gradually with time due to fatigue, corrosion and aging, or suddenly due to damage sustained in an earthquake etc. Demand resultants will change too due to changes in the structure, e.g., reduced member sectional area, or increased loads, when, e.g., larger vehicle loads become permissible.

At the network level, hazard analysis can identify $2^{n \times m}$ possible failure scenarios $S_{k}\left(k=1,2, \ldots, 2^{n \times m}\right)$ corresponding to each limit state for each bridge being exceeded or not. It can be argued that the scenarios with only one limit state exceeded for a single bridge will be characteristic of failures under everyday operating conditions due to fatigue or accidental overload. Scenarios involving simultaneous failures of multiple bridges are likely to be characteristic of significant natural disasters such as a major earthquake or flood. It can be argued that the occurrence of a failure for a given bridge and failures of other bridges are not independent in the sense of failure probability. For example, a failure of one bridge may cause another bridge to serve as an alternative route taking on increased loads and reducing its reliability. Also, particular limit state failures for the same bridge may be interdependent, e.g., foundation failure may lead to decreased superstructure reliability. However, to simplify the discussion individual probabilities are assumed independent. For each failure scenario its probability is then: 


$$
P\left(S_{k}\right)=\prod_{\substack{\text { individual limit } \\
\text { state exceeded }}} P\left(F_{p, q}\right) \times \prod_{\begin{array}{l}
\text { individual limit } \\
\text { state not exceeded }
\end{array}}\left(1-P\left(F_{p, q}\right)\right)
$$

where the first product is taken over all limit states of all bridges that fail in a given scenario, and the second product over those that do not.

The analysis of consequences assigns the probability density function of consequences for each of the scenarios. At the network level, these consequences will not be limited to the direct cost of replacement, repair or strengthening of the bridge but need to take into account indirect costs due to route closure, alternative route preparation and operations, traffic delays, impact on economy etc. The latter, indirect costs particularly if sustained for a long time may well outweigh the direct replacement costs.

Individual scenario risk can be calculated as:

$$
R_{k}=P\left(S_{k}\right) \times \mathrm{E}\left[C \mid S_{k}\right]
$$

These individual scenario risks need to be aggregated into a network level risk, $R$. In this study, the root square of sum of squares, or $L_{2}$ norm, of individual risks is used:

$$
R=\sqrt{\sum_{k=1}^{2^{n \times m}} R_{k}^{2}}=\sqrt{\sum_{k=1}^{2^{n \times m}} P^{2}\left(S_{k}\right) \times \mathrm{E}^{2}\left[C \mid S_{k}\right]}
$$

This way of aggregating individual risks emphasizes the largest risks while at the same time does not ignore the smaller risks. This can be understood as a risk prioritization. It is noted here that other risk aggregation methods are possible and, if adopted, may influence the conclusions of this discussion.

As seen in the above example, the overall network risk can be understood as consequence-weighted contributions of terms involving probabilities of individual limit state failures. Some of these failure probability terms are of the first order in the probabilities but some are products thereof. Failure probabilities are typically small numbers and so their products are negligible in comparison. For a network like the one shown in Figure 2 it is expected that consequence of simultaneous failure of two or more bridges will be larger than those of individual failures, it is, however, unlikely they will outweigh the effect of small probabilities of simultaneous failures. With that in mind, probabilities of the scenarios when only one limit state failure occurs at a time are likely to be much higher than those of multiple simultaneous failures. There are $n \times m$ such scenarios and they will be denoted by $\bar{S}_{n, m}$, while the corresponding risks $\bar{R}_{n, m}$. The aggregated risk in the network may then be simplified to:

$$
\bar{R}=\sqrt{\sum_{n, m=1}^{N, M} \bar{R}_{n, m}^{2}}=\sqrt{\sum_{n, m=1}^{N, M} P^{2}\left(\bar{S}_{n, m}\right) \times \mathrm{E}^{2}\left[C \mid \bar{S}_{n, m}\right]}
$$

This reasoning does not completely remove the low probability and high consequence events from our consideration and further comments on the matter are made when bridge failure criticality is discussed, however, we generally restrict the subsequent discussions to the individual, i.e., non-simultaneous, limit state failures. The possibility of relaxing this assumption will require more careful examination in future studies.

Assessment of the risk profile of the entire network requires information about consequences of failure scenarios and probabilities of those scenarios. Consequences of failures are related to repair or replacement costs and the disruption and changes in the traffic flow; the latter require information about traffic flows, vehicle counts and vehicular loads. Failure probabilities are, on the other hand, mostly related to the available information about structural and functional capacity and loads and other demands imposed on the structure. Even if correct in their expected values, both are typically known only with some uncertainty. In some cases, e.g., when undiscovered serious problems exist, even the mean values can be misestimated. To account for these uncertainties and errors conservative assumption must be made that increase the apparent risk. More data, and more importantly better quality and more reliable data, and information inferred from the data can reduce such uncertainties and eliminate erroneous assumptions. Thus, better estimation of risk factors in most cases reduces the risk in the first place due to typically large capacity reserves of actual structures. In some cases, when previously unknown and unexpected problems not covered by the conservativeness of less refined risk 
estimations surface, the risk may actually increase but these can be expected to be rare cases. In any case, and as the second main purpose of batter quality data, better risk estimates enable better planning for risk management including interventions such as bridge posting, repair, upgrade or replacement.

Additional data and information can augment and modify the existing knowledge via Bayesian updating. For example for demand resultants:

$$
f_{s \mid D}^{\prime \prime}=\frac{f_{s, D}^{\prime}}{f_{D}}
$$

where $f_{s \mid D}^{\prime \prime}$ is the posterior probability density function given new information $D$ is available, $f_{s \mid D}^{\prime}$ is the prior joint probability distribution function of demand resultants and new information $D$, and $f_{D}$ is the probability density function of new information $D$.

Collecting more and higher quality data increases operational costs and therefore needs to be undertaken strategically. The question is then, which failure scenarios should receive priority for enhanced data collection. Consider gradients of the overall risk with respect to individual relative changes in failure scenario probabilities and consequences:

$$
\begin{gathered}
\frac{\partial \bar{R}}{\partial P\left(\bar{S}_{k}\right) / P\left(\bar{S}_{k}\right)}=\frac{1}{\bar{R}} \times P^{2}\left(\bar{S}_{k}\right) \times \mathrm{E}^{2}\left[C \mid \bar{S}_{k}\right]=\frac{1}{\bar{R}} \times \bar{R}_{k}^{2} \\
\frac{\partial \bar{R}}{\partial E\left[C \mid \bar{S}_{k}\right] / \mathrm{E}\left[C \mid \bar{S}_{k}\right]}=\frac{1}{\bar{R}} \times P^{2}\left(\bar{S}_{k}\right) \times \mathrm{E}^{2}\left[C \mid \bar{S}_{k}\right]=\frac{1}{\bar{R}} \times \bar{R}_{k}^{2}
\end{gathered}
$$

The most efficient ways of estimating better the overall risk (including risk estimate reduction) resulting from uncertainties or errors in probabilities and/or consequence estimation, based on Eqs. (8) and (9), will target failure scenarios with highest risks. This reasoning underpins the first part of the justification of the risk and criticality based data collection strategy presented in this paper: In order to either reduce the risk and bring it below an acceptable threshold or monitor and manage the risk to ensure it never exceeds the threshold, enhanced data collection regimes should prioritize those bridges and failure scenarios that present the highest risk within the network. Bringing the cost aspect into the discussion and acknowledging that data collection costs may vary between data collection regimes and individual bridges and failure scenarios, managing risk will be based on ensuring optimal trade-offs between the benefits of risk reduction and costs required to achieve those.

The preceding considerations focused on risk as a product of failure probability and consequences. However, the area of low probability and high consequences proves to be challenging for risk assessment as in such cases risk is a small number multiplied by a large number with a somewhat "arbitrary" result. Stewart and Melchers ${ }^{10}$ see this as not a problem with the risk analysis as such but rather with the decision making based on risk assessment. However, large consequences, or impacts, or event/asset criticality are important aspects of risk perception warranting special treatment of such cases.

If we rank the consequences of individual limit state failures appearing in Eq. (6), criticality will be another criterion for prioritization of bridges and their failure types for enhanced data collection approaches. A cut-off line will have to be drawn between failure scenarios and/or bridges considered critical or not. The failure scenarios flagged in this way and not ranked highly by their risk must have low failure probabilities. Risk management focusing on criticality for such scenarios will be based on ensuring failure probabilities are kept below a certain, very stringent, level. Adoption of enhanced data collection regimes for such failure scenarios can then be rationalized by the need to accurately assess failure probabilities and monitor any changes. In other words, it can be argued that for critical failure scenarios and bridges reliability rather than risk as such needs to be managed, and in fact managed very strictly. We acknowledge here that our decision to include criticality as a criterion of risk perception is to an extent arbitrary as treatment of low probability and high consequence events vary with some sources suggesting that the action of choice could well only be "mopping up" after an event occurs ${ }^{10}$. 


\section{RISK AND CRITICALITY BASED BRIDGE DATA COLLECTION STRATEGY}

Asset managers face increasing pressures due to the high expectations of the performance and reliability of transportation networks on the one hand, and limited funding for maintenance and upgrade of bridges on the other. Infrastructure ageing, deterioration and occurrence of defects are a fact of life and it is impossible to maintain all bridges and at all times to a nearly perfect condition. Realistic and pragmatic asset management is rather about identifying, assessing and managing risks through cost effective approaches and interventions.

Advanced bridge asset management depends on the availability of quality inventory, performance and condition data, including network level data. However, data collection faces the challenge of limited resource that can be committed to the task. It is argued here that efficient data collection needs to be aligned with strategic drivers of the whole asset management cycle, i.e., risk and criticality. To that end, a stepped approach to data collection, following general asset management developments promoted by International Infrastructure Management Manual ${ }^{15}$, is proposed as succinctly outlined in Table 1. This framework proposes core, intermediate and advanced data collection development levels adopted after Paterson and Scullion ${ }^{16}$. As the criticality and/or risk of a bridge or failure scenario increases, its data collection regime will move from core through intermediate towards advanced. Guidelines about the types of data that each regime entails are provided in Bush et $\mathrm{al}^{17}$.

In this strategy, bridges on a given network will be assessed for their risk and criticality. Figure 3 shows an example of such assessment outcomes. Based on this information, bridges will be prioritized for core, intermediate or advanced data collection regime. Intermediate and advanced data collection regimes are likely to be costlier than current approaches and it is expected that the required changes in data collection need to be cost neutral. Thus, each asset manager will have the choice of drawing the core/intermediate and intermediate/advanced boundaries to suit their particular budgetary circumstances and risk tolerance.

Table 1. Risk and criticality based bridge data collection strategy.

\begin{tabular}{|l|l|l|l|}
\hline $\begin{array}{l}\text { Data collection } \\
\text { regime }\end{array}$ & $\begin{array}{l}\text { Failure risk- } \\
\text { criticality band }\end{array}$ & Assessment resolution & Data collection tools \\
\hline Core & Low & Aggregate bridge risk & $\begin{array}{l}\text { Visual inspections every 3-6 years } \\
\text { Limited, usually reactive SHM }\end{array}$ \\
\hline Intermediate & Intermediate & Individual limit state risks & $\begin{array}{l}\text { Visual inspections every 2-3 years } \\
\text { Some, reactive and proactive SHM }\end{array}$ \\
\hline Advanced & High & $\begin{array}{l}\text { Individual structural or functional } \\
\text { element risks }\end{array}$ & $\begin{array}{l}\text { Visual inspections every 1-2 years } \\
\text { Extensive, mostly proactive SHM }\end{array}$ \\
\hline
\end{tabular}

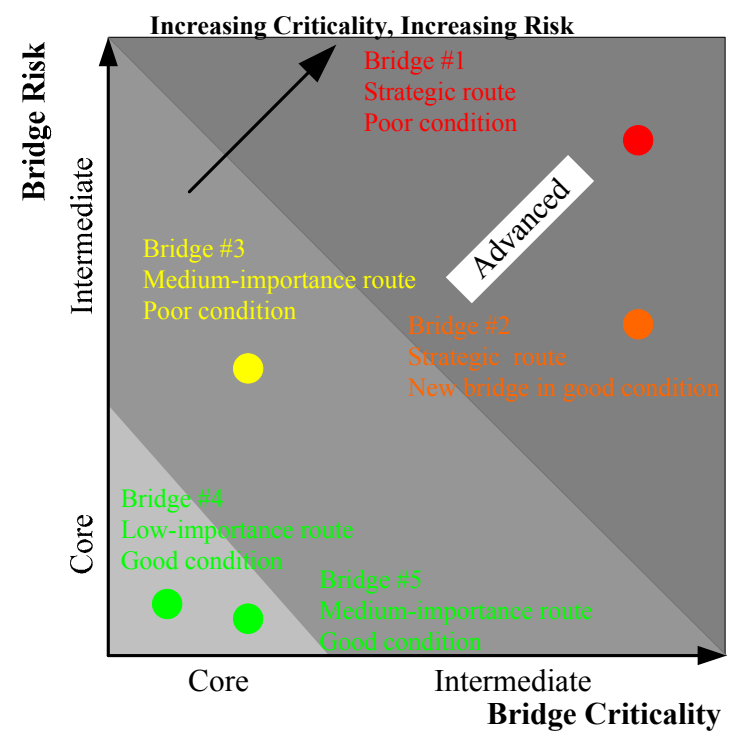

Figure 3. Illustration of risk and criticality based bridge prioritization for core, intermediate and advanced data collection regimes. 
With increased criticality and/or risk the requirements of the data collected need to be less subjective and accurate. Visual inspections have known reliability and repeatability issues ${ }^{18}$ and improvements can be achieved with a wider use of SHM.

\subsection{Core data collection regime}

Bridges for which core data collection regime applies will have both low criticality and low risk of individual limit state failures. Risk will be reported as aggregated at the bridge level. Those bridges will require a lower level of risk management, with the practices focusing on core asset management. A core asset management approach is considered appropriate for these bridges as they will be generally in overall good condition, have a limited impact on the network if the service level is reduced, and will be operating well within the operational capabilities. Based on this, only limited performance and condition data will be collected. The primary source of data collection for these bridges will be visual inspections. This is considered adequate as potential inaccuracies will not have a significant effect on risk. Visual inspections will provide key data such as condition ratings, defect descriptions, defect risk ratings, maintenance action recommendations, cost estimates and geometrical data to be used in the inventory. The frequency of visual inspection can also be reduced to 3-6 yearly, a departure from the most common biennial schedule, and resources freed in this way redirected to bridges falling into intermediate and advanced regime requirements. SHM will likely only be used sparingly and reactively in response to the greatest risks and visual inspection findings.

\subsection{Intermediate data collection regime}

If the criticality and/or risk ratings increase the data requirements change from core to intermediate. These bridges will have an increased impact on network performance, be in a poorer structural and operational state, or be structures operating close to their performance envelope. Intermediate bridges will therefore require a more accurate understanding of their risks and more accurate data collection approach. Individual limit state risks will be reported and considered in addition to the bridge level aggregate risks. In this way particular limit state risks can be prioritized for data collection rather than a whole bridge. The bridge may then have varying regimes for data collection for different limit states related to, e.g., superstructure safety and scour safety. The requirements for the intermediate regime are expected to provide sufficient data to allow advanced asset management to be carried out, such that forward planning and optimisation can be undertaken. Data will typically be collected using visual inspections on a 2-3 yearly cycle. SHM will be used reactively but also proactively as a greater level of data accuracy is required for advanced asset management. Those measures will provide lower variation on risk estimates, and therefore improved decision reliability, thereby improving short and long term planning. Further to this, network level data such as vehicle counts, truck weights, flood data, and seismic data will be collected as this is required for advanced decision making.

\subsection{Advanced data collection regime}

The advanced data collection regime is only for the most critical or high risk bridges. These bridges are likely to have a pivotal role in the operation of the network, are in a poorer state of repair, are operating close to their performance limits, or, despite being in good shape, are extremely critical. More accurate and detailed data has to be collected if the risks are to be managed appropriately and it will be cost beneficial to use more advanced techniques to collect the data. Risks will be established at individual structural element level such as for individual girders etc. Data will be collected using the full range of evaluation and monitoring techniques including frequent, 1-2 yearly visual inspections and proactively deployed SHM systems. The advanced approach therefore provides the most developed bridge management process allowing accurate short and long term planning to be undertaken. This will result in improved management of critical and high risk bridges and therefore improved network risk and functionality management.

\subsection{Application example}

In real life applications of risk analysis to bridges it is very uncommon to have detailed and refined probabilistic information about both failure probability and consequences. To deal with the lack of detailed information various scoring or similar "semi-probabilistic" schemes are employed. The scheme we use in this research was proposed by Moon et al. ${ }^{8}$ They calculate risk using the formula:

$$
R=p(H) \times p(F \mid H) \times \mathrm{E}[C \mid F] \times U
$$

where $R$ is the risk, $p(H)$ is a given hazard probability, $p(F \mid H)$ is the probability of failure given hazard occurrence, $\mathrm{E}[C \mid F]$ are the expected consequences of failure, and $U$ is the uncertainty premium that depends on the sophistication and perceived accuracy of the risk assessment method undertaken and data used. For each bridge, risks related to four

7983 - 18 V. 2 (p.8 of 13) / Color: No / Format: A4 / Date: 2011-03-03 06:41:24 PM 
broadly defined limit states, namely structural safety, hydraulic/geotechnical safety, serviceability, durability and maintenance, and functionality are calculated separately using Eq. (10) and aggregate risk for the whole bridge is given as the root square of the sum of individual risk squares. Detailed tables are provided in Moon et al. ${ }^{8}$ that enable quick calculation of the factors in Eq. (10). Rather than using probabilities as such, relative scores ranging between 1 and 3 for the first three factors are given. For example, the score for hydraulic/geotechnical hazard depends on design flood return period, seismic design category, distance from the coast, possibility of vessel impact, scour potential and history of hazards.

The uncertainty premium $U$ takes into account the sophistication and accuracy of the approaches and data used for risk analysis. Five different values are proposed, ranging from 2.5 for assessments based on minimum standard visual inspection and document review, to 1.0 when best practice visual inspection and document review are used together with best practice non-destructive evaluation and SHM. This feature of the methodology proposed by Moon et al. ${ }^{8}$ makes it very well aligned with the tiered criticality and risk based bridge data collection strategy proposed in this paper. It justifies the need for more data and the use of more accurate data collection techniques, such as SHM, for managing risks.

In the following, several bridges taken from the New Zealand state highway network are considered to illustrate the approach. Figure 4 shows photographs of the bridges. Table 2 provides a brief description of each bridge and associated hazards, vulnerabilities, failure consequences and type of data available. Table 3 shows criticality and risk assessment process and results. Note that while aggregating risks, the root mean square values were used as a modification to the method of Moon et $\mathrm{al}^{8}$. The aggregated criticality was assumed to be the largest criticality of the four scores for individual limit state consequences. Finally, Figure 5 presents graphically aggregated criticality and risks for each bridge from Table 3. The core/intermediate/advanced boundaries are indicative only as where exactly these are drawn will be left to the asset manager's discretion. Of particular interest is the fact that application of best practice data collection including SHM significantly lowers the risk for Bridge \#3 (Auckland Harbor Bridge); if minimum standard visual inspection only were used the aggregate risk would be nearly 2.5 times higher.

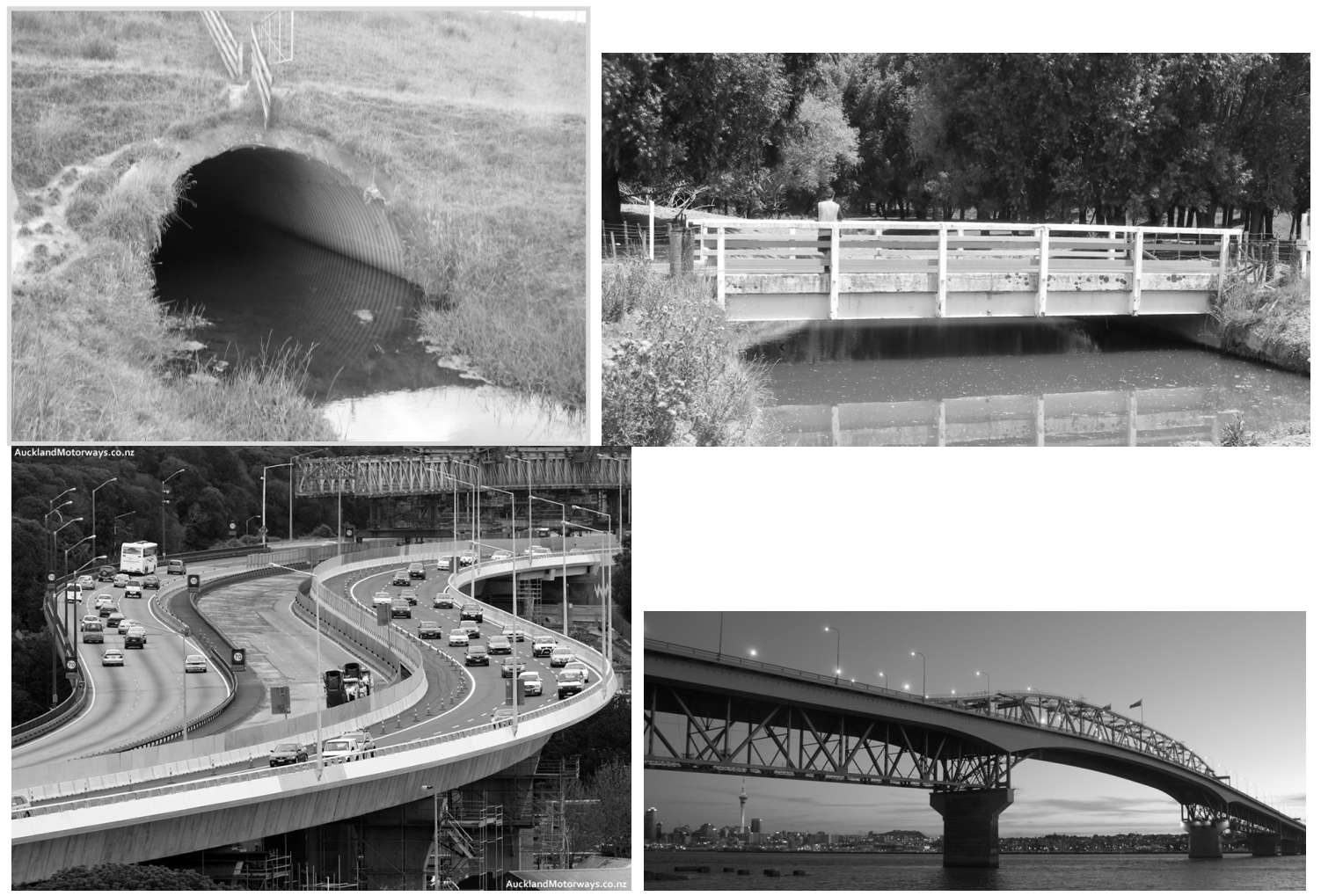

Figure 4. Analyzed bridges (clockwise from top left): Bridge \#1: Corrugated steel culvert, Bridge \#2: Single span timber bridge, Bridge \#3: Auckland Harbor Bridge (AHB), Bridge \#4: Newmarket Viaduct (AuclandMotorways.co.nz). 
Table 2. Analyzed bridges and their descriptions.

\begin{tabular}{|c|c|}
\hline Bridge \# & Description \\
\hline $\begin{array}{l}\text { Bridge \#1: } \\
\text { Corrugated steel culvert }\end{array}$ & $\begin{array}{l}4 \mathrm{~m} \text { diameter corrugated steel culvert supports state highway of national } \\
\text { strategic importance } \\
\text { Overall good condition; only minor corrosion to the barrel; no scour } \\
\text { Replacement cost low; annual average daily traffic (AADT) }>50,000 \\
\text { vehicles; heavy commercial vehicle 5\%; reasonable level of service could be } \\
\text { restored within a few days; temporary measures quickly available; } \\
\text { alternatives available, with only minor reductions to service level } \\
\text { Data collected via regular minimum standard visual inspections }\end{array}$ \\
\hline $\begin{array}{l}\text { Bridge } \# 2 \text { : } \\
\text { Single span timber bridge }\end{array}$ & $\begin{array}{l}12 \mathrm{~m} \text { span timber bridge carrying road of local importance over a small river; } \\
\text { Designed to outdated load standards; overall moderate condition } \\
\text { Replacement cost moderate between NZ } \$ 100 \mathrm{~K} \text { and NZ } \$ 1 \mathrm{~m} \text {; AADT is } 1000 \text {; } \\
\text { service can be returned after several days, with a temporary bridge installed } \\
\text { to cross the river } \\
\text { Data collected via regular minimum standard visual inspections }\end{array}$ \\
\hline $\begin{array}{l}\text { Bridge \#3: } \\
\text { Auckland Harbor Bridge } \\
\text { (AHB) }\end{array}$ & $\begin{array}{l}\text { Key link across a harbor supporting state highway of national strategic importance at } \\
\text { the heart of the major economic centre of New Zealand; complex truss bridge with } \\
\text { "clip-on" extensions on both sides } \\
\text { Navigable shipping channel; coastal environment } \\
\text { Known fatigue issues in extensions (heavy vehicles prohibited on extensions); } \\
\text { extensions recently strengthened but only limited service life expected } \\
\text { Replacement cost very high; AADT for extensions }>38,000 \text {, centre truss } \\
>80,000 \text {; major service would take }>1 \text { year to restore; a detour available for } \\
\text { long routes, but nothing available locally; failure will cause significant delays } \\
\text { in the region and impact heavily on local, regional, and inter-regional } \\
\text { commerce; a national icon } \\
\text { Individual management plan implemented including best practice visual } \\
\text { inspections, NDE, and SHM }\end{array}$ \\
\hline $\begin{array}{l}\text { Bridge } \# 4 \text { : } \\
\text { Newmarket Viaduct }\end{array}$ & $\begin{array}{l}\text { Key link supporting state highway of national strategic importance at the heart of the } \\
\text { major economic centre of New Zealand; completed in } 2011 \text {; twin post-tensioned } \\
\text { bridges with } 12 \text { spans } \sim 60 \mathrm{~m} \text { each } \\
\text { Replacement cost very high }>\text { NZ } \$ 200 \mathrm{M} \text {; AADT }>160,000 \text {; service would } \\
\text { take }>1 \text { year to restore; detours available but failure will cause significant } \\
\text { delays in the region and impact heavily on local, regional, and inter-regional } \\
\text { commerce; a national icon } \\
\text { Data collected via best practice visual inspections and technical analyses conducted; a } \\
\text { University-operated SHM system that could be integrated into management plan }\end{array}$ \\
\hline
\end{tabular}

\section{THE ROLE OF SHM IN RISK AND CRITICALITY BASED BRIDGE DATA COLLECTION STRATEGY}

\subsection{SHM as a tool for better assessment of consequences}

At the network level, the possible consequences of bridge failure whose assessment may be facilitated by using SHM are those related to the disruption and changes in the traffic flow. Here SHM systems will comprise sensors for monitoring traffic flows, vehicle counts and vehicular loads and may include cameras, vehicle count and classification systems, weight-in-motion, satellite images, GPS and other. It is noted that those sensors may actually be deployed far away from the bridges as determined by network traffic flow analyses and in this sense require adoption of a broader than the traditional concept of SHM. Together with network operations models and economic loss models data from the sensors will enable more accurate failure consequence predictions. Particularly useful will be data from previous disruptive events sustained by the system which, e.g., engaged alternative routes. The vast majority of risk studies will consider existing networks where some prior, often considerable, knowledge about the system risks and operations exists. With 
Table 3. Risk and criticality assessment of analyzed bridges.

\begin{tabular}{|c|c|c|c|c|c|c|c|c|}
\hline \multirow[t]{2}{*}{ Limit state } & \multicolumn{2}{|c|}{$\begin{array}{l}\text { Bridge \#1: } \\
\text { Culvert }\end{array}$} & \multicolumn{2}{|c|}{$\begin{array}{l}\text { Bridge \#2: } \\
\text { Timber bridge }\end{array}$} & \multicolumn{2}{|c|}{$\begin{array}{l}\text { Bridge \#3: } \\
\text { AHB }\end{array}$} & \multicolumn{2}{|c|}{$\begin{array}{l}\text { Bridge \#4: } \\
\text { Newmarket } \\
\text { Viaduct }\end{array}$} \\
\hline & Risk & Cons. & Risk & Cons. & Risk & Cons. & Risk & Cons. \\
\hline Structural safety & 10.0 & 2 & 7.5 & 1 & 27.0 & 3 & 11.3 & 3 \\
\hline Hydraulic/geotech. safety & 10.0 & 2 & 5.0 & 1 & 22.5 & 3 & 3.8 & 3 \\
\hline Serviceability & 5.0 & 1 & 5.0 & 1 & 12.0 & 2 & 7.5 & 2 \\
\hline Functionality & 15.0 & 2 & 5.0 & 1 & 18.0 & 2 & 7.5 & 2 \\
\hline $\begin{array}{l}\text { Aggregate: risk (RMS) } \\
\text { criticality (max cons.) }\end{array}$ & 10.6 & $\underline{2}$ & 5.7 & 1 & $\underline{20.6}$ & $\underline{3}$ & $\underline{8.0}$ & $\underline{3}$ \\
\hline
\end{tabular}

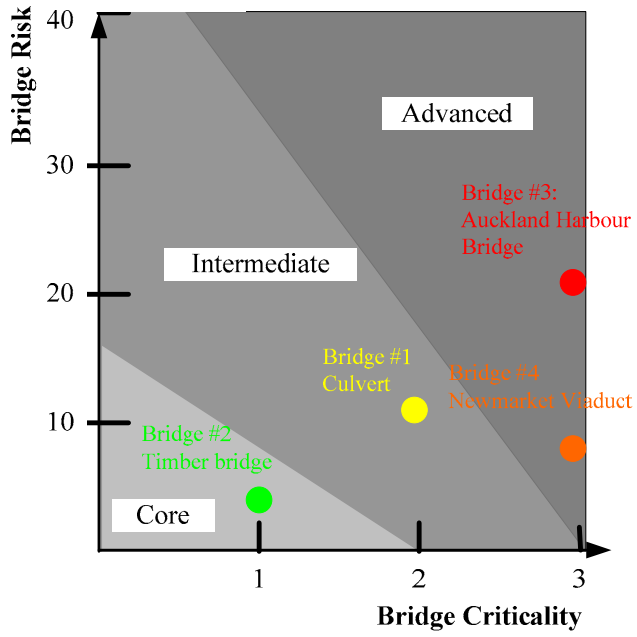

Figure 5. Risk and criticality assessment of analyzed bridges.

careful examination, additional SHM information can augment and modify this existing knowledge via Bayesian updating.

\subsection{SHM as a tool for better assessment of failure probability}

This is mostly the area which is concerned with sensors deployed on the bridge or within its immediate vicinity. These sensors measure loads and environmental effects (temperature, humidity, solar radiation) on the structure and structural responses. However, it is noted here that other, "wide-area" monitoring systems may be integrated in to bridge data collection regime. Those systems include seismic monitoring arrays, weather and hydraulic river flow monitoring arrays, tidal flow and tsunami monitoring systems and other.

Again, if functional and operational aspects of bridge risk are to be managed using better data, there is need to looks outside the sensors measuring loads and structural responses. Other aspects will require their own data and these include traffic flows over the bridge, and demands on and responses of non-structural components such as tire pressures acting on pavements and their deformations and deterioration.

Chen ${ }^{19}$ argues one of the main the reasons why SHM techniques have not yet found widespread applications in bridge asset management is misalignment of the significant portion of SHM research outcomes with the real needs of the industry. This is because SHM research as applied to bridges and other structural systems has not freed itself from the legacy of the vibration based SHM focus prevailing in the machine and aerospace industries. There are, however, specific issues responsible for bridge failures where SHM can make a real positive difference, including ${ }^{19,20}$ :

- Scour rate, 
- Reinforcement steel corrosion,

- Delaminations in concrete decks,

- Crack width in concrete structures,

- Fatigue in steel structures, and

- Large and/or yielding strains in steel.

As discussed in this paper, aligning bridge data collection approaches, including SHM, with the risk focused drivers of bridge asset management and targeting the most widespread problems such as those listed above will assist with wider proactive adoption of SHM into practice.

\section{CONCLUSIONS}

This discussion paper interprets the collection of data on bridge condition and performance in the context of managing risks in the overall transportation network. Recognizing that appropriate risk management is the primary driver behind the management of transportation network assets, including bridges, the paper demonstrates how more detailed and reliable data can reduce uncertainties and be therefore used for reducing bridge and network level risks. By aggregating individual bridge risks at the network level it is shown that bridges with higher risk should receive priority for enhanced data collection. From the point of view of risk perception, another way of prioritizing, based on bridge failure consequences or criticality, is also required. These considerations lead to the formulation of a risk and criticality based bridge data collection strategy. In this strategy, three data collection regimes, namely core, intermediate and advanced, are proposed. As the criticality and/or risk of a bridge increase the structure is moved towards higher data collection levels that entail gathering of more detailed and reliable data. The higher data collection regimes rely on proactive and planned integration of SHM systems for risk management and some comments about the need to align SHM with most common bridge problems are provided. Given the network level nature of many bridge risks and criticality the need for appropriate data regarding network performance, also collected via monitoring systems, is emphasized. An example of bridge classification for data collection is provided using several existing highway bridges and simple risk scoring methodology to illustrate the discussion.

\section{REFERENCES}

1. Aktan, A. E., Catbas, F. N., Grimmelsman, K., Pervizpour, M., Curtis, J., Shen, K., Qin, X. and Cinar, O. A., "Health monitoring for effective management of infrastructure," 1st European Workshop on Structural Health Monitoring, 1245-1253 (2002).

2. Inaudi, D. and Walder, R., "Integrated structural health monitoring systems for high-rise buildings," Proceedings of the1st Middle East Conference on Smart Monitoring, Assessment and Rehabilitation of Civil Structures SMAR 2011, 1-8 (2011).

3. Abdelrazaq, A., "Validating the structural behavior and response of Burj Khalifa: Synopsis of the full scale structural health monitoring programs," Proceedings of the1st Middle East Conference on Smart Monitoring, Assessment and Rehabilitation of Civil Structures SMAR 2011, 1-18 (2011).

4. Tozser, O., Elliott, J. and Garcia, R., "Acoustic monitoring used to manage the life of cable supported bridges," Proceedings of the1st Middle East Conference on Smart Monitoring, Assessment and Rehabilitation of Civil Structures SMAR 2011, 1-8 (2011).

5. British Standards Institution, PAS 55-1:2008: Specification for the optimized management of physical assets. 2008, British Standards Institution: London.

6. Faber, M. H. and Stewart, M. G., "Risk assessment for civil engineering facilities: Critical overview and discussion," Reliability Engineering and System Safety 80 (2), 173-184 (2003).

7. Beamish, M., Tindall, P. and Billings, I., "Auckland harbor bridge fatigue assessment," Proceedings of the 2006 Austroads Bridge Conference, 1-10 (2006).

7983 - 18 V. 2 (p.12 of 13) / Color: No / Format: A4 / Date: 2011-03-03 06:41:24 PM

SPIE USE:

DB Check,

Prod Check, Notes: 
8. Moon, F. L., Laning, J., Lowdermilk, D. S., Chase, S., Hooks, J. and Aktan, A. E., " Proceedings of SPIE Conference on Nondestructive Characterization for Composite Materials, Aerospace Engineering, Civil Infrastructure, and Homeland Security 2009, 72940M-1 - 72940M-11 (2009).

9. Matousek, M. and Schneider, J., Untersuchungen zur Struktur des Zicherheitproblems bei Bauwerken, Institut für Baustatik und Konstruktion der ETH Zürich, Zürich (1977).

10. Stewart, M. G. and Melchers, R. E., Probabilistic risk assessment of engineering systems, Chapman and Hall, London (1997).

11. Frangopol, D. M., Strauss, A. and Kim, S., "Bridge reliability assessment based on monitoring," Journal of Bridge Engineering 13 (3), 258-270 (2008).

12. Orcesi, A. D. and Frangopol, D. M., "Optimization of bridge maintenance strategies based on structural health monitoring information," Structural Safety 33 (1), 26-41

13. Standards Australia, AS/NZS 4360. Risk management, Standards Australia, Sydney (2004).

14. Melchers, R. E., Structural reliability analysis and prediction, Wiley, Chichester (1999).

15. NAMS Group, International infrastructure management manual, NAMS Group, Wellington ( 2006).

16. Paterson, W. D. O. and Scullion, T., Information systems for road management: Draft guidelines on system design and data issues, World Bank, (1990).

17. Bush, S., Omenzetter, P., Henning, T. and McCarten, P., Data collection and monitoring strategies for New Zealand road bridge management, New Zealand Transport Authority, Wellington (2011), (in preparation).

18. Phares, B. M., Rolander, D. D., Graybeal, B. A. and Washer, G. A., "Reliability of visual bridge inspection," Public Roads 64 (5), 22-29 (2001).

19. Chen, G., "A pragmatic and innovative approach for civil infrastructure management - structural behavior monitoring," Proceedings of the SPIE's 17th Annual International Symposium on Smart Structures and Materials/SPIE's 15th Annual International Symposium on NDE for Health Monitoring and Diagnostics, 7649: I-1 - I-9 (2010).

20. Ansari, F., "Autonomous multi-scale self-regenerative systems for monitoring of civil structures," Proceedings of the 4th International Conference on Structural Health Monitoring of Intelligent Infrastructure, 1-7 (2009). 